\title{
Effect of Pregabalin on Postcraniotomy Pain in Patients Undergoing Supratentorial Tumor Surgery: A Randomized, Double-Blind, Placebo-Controlled Trial
}

\author{
Ritesh Lamsal ${ }^{1}$ Charu Mahajan Vikas Chauhan \\ ${ }^{1}$ Department of Neuroanaesthesiology and Critical Care, All India \\ Institute of Medical Sciences, New Delhi, India
}

Nidhi Gupta ${ }^{1} \quad$ Nitasha Mishra $\quad$ Girija Prasad Rath ${ }^{1}$

J Neurosci Rural Pract 2019;10:641-645

\begin{abstract}
Address for correspondence Charu Mahajan, MD, DM, Department of Neuroanaesthesiology and Critical Care, Neurosciences Centre, All India Institute of Medical Sciences, New Delhi 110 029, India (e-mail: charushrikul@gmail.com).
\end{abstract}

\begin{abstract}
Keywords

- analgesia

- craniotomy

- fentanyl

- postoperative pain

- pregabalin

- visual analog scale
\end{abstract}

Background and Objectives Suboptimal management of postcraniotomy pain causes sympathetic and hemodynamic perturbations, leading to deleterious effects on the neurological system and overall patient outcome. Opioids are the mainstay of postoperative pain management but have various problems when given in high doses, or for prolonged durations in neurosurgical patients. The ideal method of pain control following craniotomy generally relies on a combination of various drugs. Oral pregabalin may be an attractive alternative in these patients.

Materials and Methods Sixty, American Society of Anesthesiologists class I and II patients posted for elective supratentorial craniotomy, aged 18 and 60 years, were randomly assigned into three groups of 20 each to receive oral placebo (Group A), pregabalin $75 \mathrm{mg}$ (Group B), or pregabalin $150 \mathrm{mg}$ (Group C) before the induction of anesthesia. At the end of the surgery, patient-controlled analgesia was started with intravenous fentanyl. Visual analog scale (VAS) score was recorded every 2 hours for 24 hours, along with total postoperative fentanyl requirement.

Results There were no differences in sex, duration of surgery or anesthesia and total intraoperative fentanyl administered among the three groups. The median postoperative VAS score (Group A-18.0, Group B-20, and Group C-22.0; $p=0.63$ ) was similar in all the groups. However, postoperative fentanyl requirement over 24 hours was least in the group that received $150 \mathrm{mg}$ pregabalin (Group A-190 $\mu \mathrm{g}$, Group B-240 $\mu \mathrm{g}$, and Group $C-100 \mu \mathrm{g} ; \mathrm{p}=0.03)$.

Conclusions Even though pain scores were not significantly different, patients receiving $150 \mathrm{mg}$ oral pregabalin required the least amount of postoperative opioids.

\section{Introduction}

The paradigm that craniotomy is associated with minimal postoperative pain is an age-old belief that has often led to inadequate analgesia in postcraniotomy patients. Pain can elicit a complex set of neural, cardiac, and respiratory changes that can lead to increased cerebral blood flow and intracranial pressure, cerebral edema, and even intracranial bleeding. Many patients may suffer from debilitating chronic pain syndromes.
Pregabalin is a structural analog of gamma-aminobutyric acid with analgesic, anticonvulsant, and anxiolytic effects. ${ }^{1}$ It is approved for treating neuropathic pain and used in diabetic peripheral neuropathy, postherpetic neuralgia, and central or peripheral neuropathic pain syndromes. Even though pregabalin has been evaluated for postoperative analgesia in spine surgery, ${ }^{2}$ laparoscopic hysterectomy, ${ }^{3}$ laparoscopic cholecystectomy, ${ }^{4}$ orthopaedic surgery, ${ }^{5}$ thoracotomy, ${ }^{6}$ and day-care gynecological laparoscopic surgery, ${ }^{7}$ there is a
License terms

10.1055/s-0039-3399490

ISSN 0976-3147. 
paucity of trials investigating its effects in attenuating postoperative pain after intracranial surgeries. This study was designed to evaluate the effect of preoperative pregabalin on postoperative pain and analgesic requirement in patients undergoing supratentorial craniotomy.

\section{Materials and Methods}

This randomized, double-blind, placebo-controlled study was conducted after approval from the institute's ethics committee. Written informed consent was obtained from all the patients. Sixty patients posted for elective supratentorial craniotomy for tumor resection, between the ages of 18 and 65 years, American Society of Anesthesiologists physical status I and II and with Glasgow Coma Scale of 15, were included in the study. Patients with hepatic or renal diseases, mental disability, neurological deficits (precluding their use of a patient-controlled analgesia [PCA] device), large tumors ( $>50 \mathrm{~mm}$ in any dimension), and known allergy to the study medication or history of substance abuse were excluded from the study. A thorough general examination was performed 1 day prior to the surgery, and all significant details were noted. Patients meeting the inclusion criteria during the preanesthetic evaluation were explained about the use of PCA device and the $100-\mathrm{mm}$ visual analog scale (VAS) for pain ( 0 = no pain; 100 = worst imaginable pain). Eligible patients were then randomly assigned to three groups of twenty each, with the help of a computer-generated table of random numbers, to receive oral placebo (Group A), pregabalin $75 \mathrm{mg}$ (Group B), or pregabalin $150 \mathrm{mg}$ (Group C). The patient and the observer were blinded to the three study groups. The drug containing envelope was prepared by a person who was not involved in this study. Each patient was given one envelope by a staff nurse who was unaware of the study. The patient was asked to take the capsule with a sip of water 1 hour before the induction of anesthesia.

Standard monitors included electrocardiography, pulse oximetry $\left(\mathrm{SpO}_{2}\right)$, noninvasive blood pressure (NIBP), and capnography. General anesthesia was induced with propofol 1 to $2 \mathrm{mg} / \mathrm{kg}$ and fentanyl $2 \mu \mathrm{g} / \mathrm{kg}$ to achieve loss of response to verbal commands. Muscle relaxation was achieved with vecuronium $0.1 \mathrm{mg} / \mathrm{kg}$. After tracheal intubation, anesthesia was maintained with nitrous oxide in oxygen (60:40) and isoflurane with a combined minimal alveolar concentration of 1.0 to 1.2. Fentanyl and vecuronium were supplemented as per the discretion of the consultant anesthesiologist. Radial artery cannulation was performed in all the patients for invasive blood pressure monitoring. Total intraoperative fentanyl used, duration of surgery and anesthesia were recorded. At the end of the surgery, residual neuromuscular blockade was reversed with neostigmine and glycopyrrolate. After tracheal extubation, all patients were shifted to the neurosurgical intensive care unit (NSICU). Assessment of pain, NIBP, heart rate, $\mathrm{SpO}_{2}$ and respiratory rate were done on arrival in the NSICU. Following this, patients were given a bolus of fentanyl $1 \mu \mathrm{g} / \mathrm{kg}$ through the PCA pump. The incremental dose was set at 0.25 to $0.5 \mu \mathrm{g} / \mathrm{kg}$ with a lock-out interval of 10 minutes and 4-hour limit of $0.4 \mathrm{mg}$. No background infusion of fentanyl was given. No other supplemental analgesic drugs or local anesthetics were given. VAS scores $(100 \mathrm{~mm})$ were recorded every 2 hours for the next 24 hours. A single observer who was blinded to the groups recorded all the measurements including severity of postoperative pain and postoperative fentanyl requirement. Side effects, if any, such as sedation, respiratory depression, pruritus, and nausea/vomiting, were recorded. In the event of any major problem (uncontrolled pain, sedation, obtundation, and severe respiratory depression), the PCA device was discontinued.

A sample size of 60 was chosen as a convenient number for this study based on feasibility issues of conducting this study at our institute. All statistical tests were performed using IBM SPSS Statistics, version 21 for Windows (IBM Corporation, New York, United States). Categorical variables such as sex were compared between the groups using the Pearson's chi-squared test. Continuous variables were first assessed for normality of distribution. Baseline intraoperative continuous variables were then appropriately compared among the three groups using the Kruskal-Wallis test. The outcome variables including 24-hour VAS scores and 24-hour postoperative fentanyl requirement were expressed as median and range (interquartile range) and compared using the Kruskal-Wallis one-way analysis of variance. A $p$-value $<0.05$ was considered statistically significant.

\section{Results}

A total of 72 patients were assessed for eligibility (- Fig. 1). Among these, 12 patients were excluded for not meeting the inclusion criteria, leaving 60 patients for randomization into three groups. Five patients were either not extubated or reintubated before 24 hours. These included two patients each in Group A and Group B and one patient in Group C. Scoring of pain and the use of PCA pump were not possible in these patients and they were excluded from the final analysis.

The median age of the patients in Group $C$ was slightly lower compared with that of Groups A and B $(p=0.01)$ (-Table 1). Males and females were evenly distributed $(p=0.77)$ in the three groups. There was no statistically significant difference in the duration of surgery $(p=0.72)$, duration of anesthesia $(p=0.96)$, or total intraoperative fentanyl administered $(p=0.21)$ among the three groups of patients (-Table 1$)$. In Group A, one patient required reintubation for pneumocephalus and another patient developed aphasia. In Group B, one patient was electively ventilated in the postoperative period for tense brain and another patient required reintubation in the ICU for seizures. In Group C, one patient was electively ventilated postoperatively for tense brain. These five patients were excluded from the final analysis. None of the patients experienced any side effects due to the study drug.

The median (interquartile range) VAS scores over 24 hours were 18 (16) in Group A, 20 (15) in Group B, and 22 (16) in Group C (-Table 2). This difference was not statistically significant $(p=0.63)$. The requirement of postoperative fentanyl over 24 hours was least in Group C: 100.0 (130) $\mu$ g, compared with 190.0 (365.0) $\mu \mathrm{g}$ in Group A and 240.0 (112.5) $\mu \mathrm{g}$ in Group B (-Fig. 2). This difference was statistically significant $(p=0.03)$ (-Table 2). 


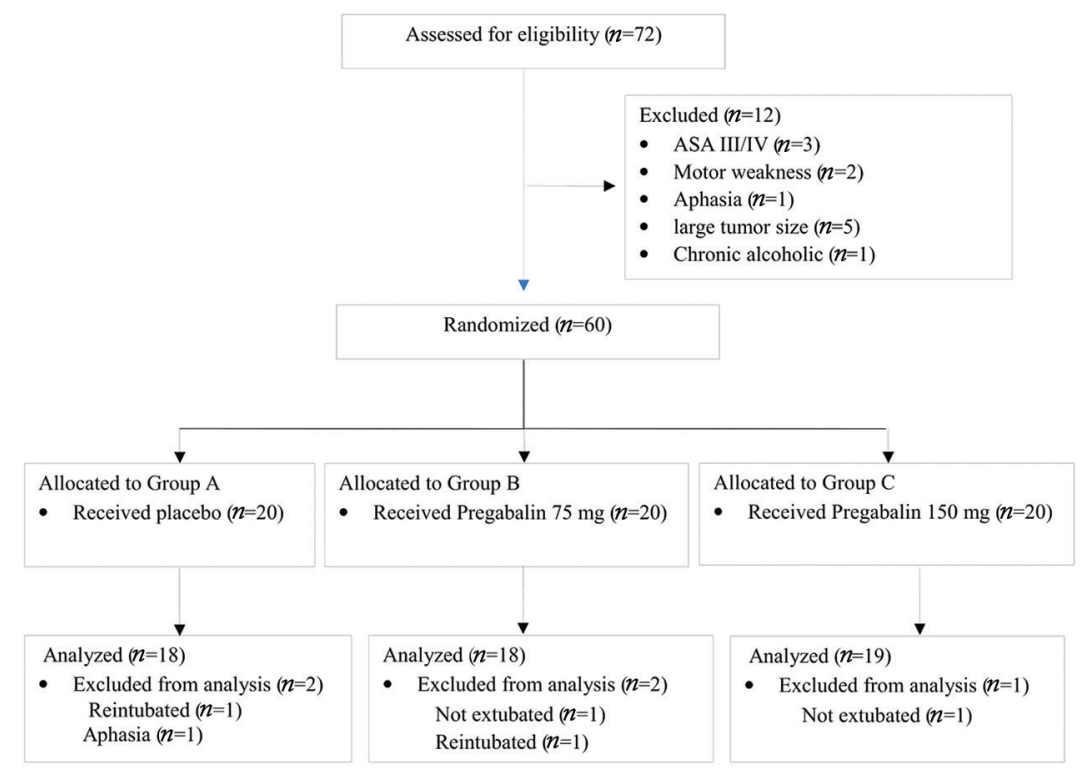

Fig. 1 CONSORT diagram. ASA III/IV, American Society of Anesthesiologists Class III/IV.

Table 1 General characteristics

\begin{tabular}{|l|l|l|l|l|}
\hline Variable & Group A $(\boldsymbol{n}=18)$ & Group B $(\boldsymbol{n}=18)$ & Group C $(\boldsymbol{n}=19)$ & $\boldsymbol{p}$-Value \\
\hline Age $(\mathrm{y})$ & $35,20(18-65)$ & $49,19(20-60)$ & $30,11(19-57)$ & 0.01 \\
\hline Weight $(\mathrm{kg})$ & $61,15(35-90)$ & $60,7(43-81)$ & $62,17(45-80)$ & 0.38 \\
\hline Sex (male/female) & $14 / 6$ & $15 / 5$ & $16 / 4$ & 0.77 \\
\hline Surgery duration (min) & $248,143.8(105-420)$ & $240,75(140-560)$ & $245,101(90-600)$ & 0.72 \\
\hline Anesthesia duration $(\mathrm{min})$ & $342,191.3(210-540)$ & $317.5,77.5(180-620)$ & $300,145(150-720)$ & 0.96 \\
\hline Intraoperative fentanyl $(\mu \mathrm{g})$ & $275.0,187.5(100-475)$ & $300.0,100.0(225-500)$ & $300.0,150.0(100-1000)$ & 0.21 \\
\hline
\end{tabular}

Note: Data presented as median, interquartile range (range), or $n^{\text {a }}$. Group A: placebo, Group B: pregabalin $75 \mathrm{mg}$, Group C: pregabalin $150 \mathrm{mg}$.

Table 2 Postoperative pain parameters $(24 \mathrm{~h})$

\begin{tabular}{|l|l|l|l|l|}
\hline 24-h details & Group A $(\boldsymbol{n}=18)$ & Group B $(\boldsymbol{n}=18)$ & Group C $(\boldsymbol{n}=19)$ & $\boldsymbol{p}$-Value \\
\hline VAS score & $18,16(10-40)$ & $20,15(22-33)$ & $22,16(25-35)$ & 0.63 \\
\hline Postoperative fentanyl $(\mu \mathrm{g})$ & $190.0,365.0(50-1539)$ & $240.0,112.5(100-500)$ & $100.0,130.0(30-400)$ & 0.03 \\
\hline
\end{tabular}

Abbreviation: VAS, 100-mm visual analog scale.

Note: Data were presented as median and range (interquartile range); Group A: placebo, Group B: pregabalin 75 mg, Group C: pregabalin 150 mg.

\section{Discussion}

The severity of craniotomy pain can be significant, and more importantly, many patients may not get satisfactory pain relief because adequate analgesia is not prescribed. An old British survey more than 20 years back found that, in contrary to the widespread belief, moderate-to-severe pain is common after craniotomy and the pain responds very poorly to codeine. ${ }^{8}$ Yet, as recent as 2009, over two-third of the physicians in the United Kingdom still use codeine or dihydrocodeine as the first-line analgesic following craniotomy, using more potent opioids such as morphine, very sparingly. ${ }^{9}$ Opioids have been associated with undesirable side effects such as postoperative nausea and vomiting, ${ }^{10}$ respiratory depression, ${ }^{11}$ excessive sedation, ${ }^{12}$ neuropsychiatric manifestations, ${ }^{13}$ dizziness, ${ }^{14}$ and visual disturbances. ${ }^{14}$ Furthermore, there are concerns regarding its effects on cerebral hemodynamics and intracranial pressure. ${ }^{15,16}$ This has led to the widespread reservation about opioid use in neurosurgical patients, encouraging investigators to explore the effects of novel agents such as ketamine, gabapentinoids, and dexmedetomidine.

Gabapentinoids have been studied for postoperative pain relief after surgeries under general as well as regional anesthesia. ${ }^{17}$ There are several studies on general surgeries that have demonstrated that even a single preoperative dose of gabapentinoids can reduce postoperative pain. Agarwal et $\mathrm{al}^{4}$ found that preoperative oral pregabalin $150 \mathrm{mg}$ was effective and safe for reducing both postoperative pain and fentanyl requirement in patients undergoing laparoscopic 


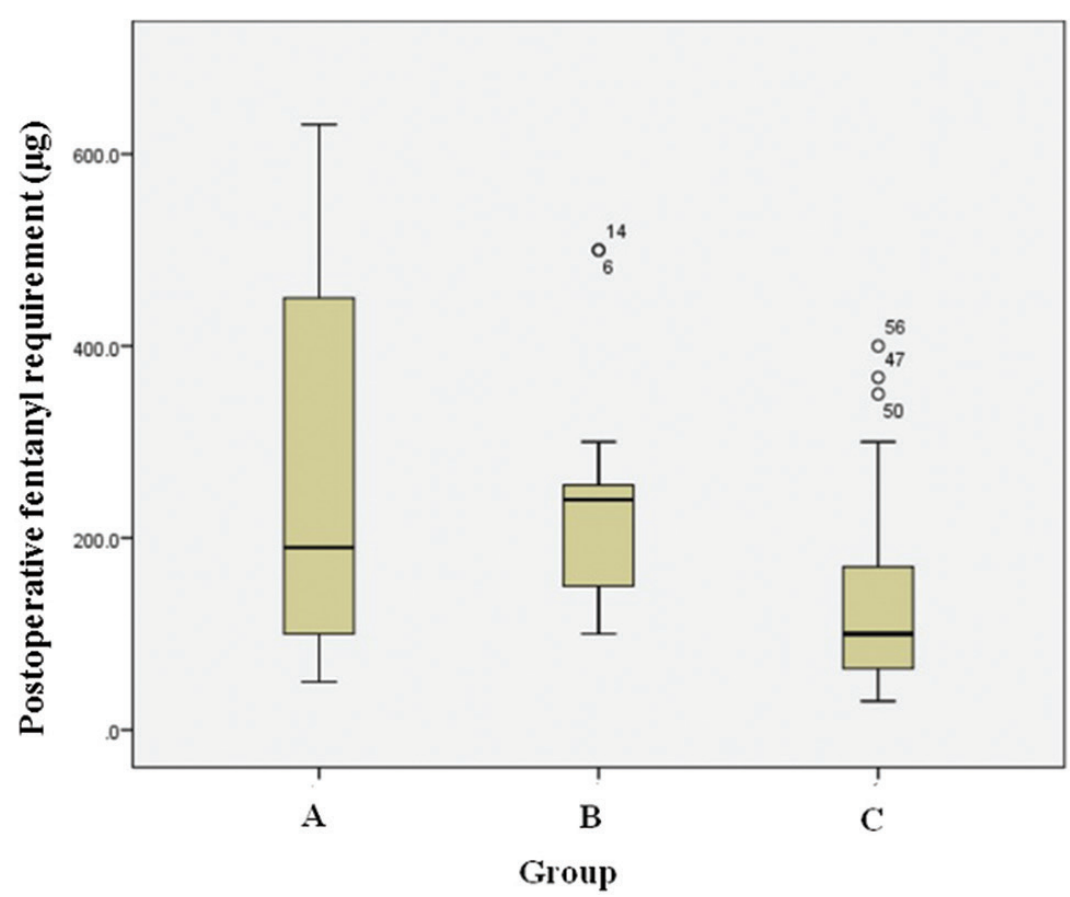

Fig. 2 24-Hour postoperative fentanyl consumption.

cholecystectomy. On the other hand, even though there was no improvement in VAS scores with pregabalin use in our study, there was a significant reduction in postoperative fentanyl requirement in those receiving $150 \mathrm{mg}$ pregabalin. It is possible that patients undergoing neurosurgery behave differently than general surgical patients and that prolonged duration of neurosurgical procedures can reduce the analgesic effect of a single low dose $(75 \mathrm{mg})$ of preoperative pregabalin (elimination half-life: $5.5-6.5$ hours).

A meta-analysis also found that analgesic effect of pregabalin varied with the type of surgery-most apparent with short-duration gynecologic, laparoscopic, and ENT surgeries, with no clear benefit in some other types of surgeries..$^{18}$ It is also established that preoperative oral pregabalin when given in doses up to $150 \mathrm{mg}$ is safe and does not cause increase in postoperative sedation. ${ }^{12}$ Mishriky et al performed a meta-analysis in which they found that pregabalin not only improves postoperative analgesia but also causes increased sedation, dizziness, and visual disturbances. ${ }^{19}$ The authors studied the effect at different doses and regimens and found that the risk of sedation increased when pregabalin $300 \mathrm{mg}$ was used as either single or multiple doses. However, we found no side effects associated with doses used in our study.

Studies of pregabalin in neurosurgical patients are scarce. There is only one published randomized study of pregabalin use for analgesia in patients undergoing craniotomy by Shimony et al. ${ }^{20}$ This study found that pregabalin attenuated pain scores and reduced analgesic consumption in postcraniotomy patients. Even though our findings are similar, their study design was very different as patients were given two doses of oral pregabalin $(150 \mathrm{mg}$ ) before surgery, which they continued to receive postoperatively twice daily for 3 days. There may be some evidence to suggest that prolonged dosing protocols of pregabalin have better analgesic effects, but it is difficult to justify such protracted regimens in neurosurgical patients, where potential side effects such as undue sedation, dizziness, vomiting, and visual disturbances can mask the ongoing neurologic problems. Such extended pregabalin regimens for analgesia are unlikely to be adopted by most NSICUs. Our pregabalin dosing protocol using a single dose of $150 \mathrm{mg}$ is extremely convenient to administer, is cost-effective, and is devoid of any major intraoperative or immediate postoperative side effects. Importantly, it reduces the requirement of postoperative opioids, mitigating some of the major concerns of using opioids in higher doses in neurosurgical patients.

It was beyond our scope to study the long-term effects of pregabalin. The limited sample size of the study did not permit data stratification based on the duration of the surgery, location and invasiveness of the tumor, the size of the craniotomy wound, or the degree of tissue dissection, all of which may have confounded the data. Larger trials, especially those employing different dose and duration protocols, and evaluating long-term outcome, are warranted to shed more light on the topic. The findings of this study can pave the way for designing such trials in neurosurgical patients in future.

\section{Conclusions}

Single oral dose of pregabalin $75 \mathrm{mg}$ has no effect in lowering 24-hour mean VAS score or postoperative fentanyl requirement in patients undergoing supratentorial craniotomy. However, the findings of this study support the use of $150 \mathrm{mg}$ pregabalin for preemptive analgesia before routine supratentorial tumor surgeries to reduce the requirement of postoperative opioids, unless specific contraindications for the use of the drug exist. 


\section{Funding}

The study was financially supported by the All India Institute of Medical Sciences, New Delhi, India.

\section{Conflict of Interest}

None declared.

\section{References}

1 Ben-Menachem E. Pregabalin pharmacology and its relevance to clinical practice. Epilepsia 2004;45(Suppl 6):13-18

2 Gianesello L, Pavoni V, Barboni E, Galeotti I, Nella A. Perioperative pregabalin for postoperative pain control and quality of life after major spinal surgery. J Neurosurg Anesthesiol 2012;24(2):121-126

3 Jokela R, Ahonen J, Tallgren M, Haanpää M, Korttila K. A randomized controlled trial of perioperative administration of pregabalin for pain after laparoscopic hysterectomy. Pain 2008;134(1-2):106-112

4 Agarwal A, Gautam S, Gupta D, Agarwal S, Singh PK, Singh U. Evaluation of a single preoperative dose of pregabalin for attenuation of postoperative pain after laparoscopic cholecystectomy. Br J Anaesth 2008;101(5):700-704

5 YaDeau JT, Lin Y, Mayman DJ, et al. Pregabalin and pain after total knee arthroplasty: a double-blind, randomized, placebo-controlled, multidose trial. Br J Anaesth 2015;115(2):285-293

6 Yoshimura N, Iida H, Takenaka M, et al. Effect of postoperative administration of pregabalin for post-thoracotomy pain: a randomized study. J Cardiothorac Vasc Anesth 2015;29(6):1567-1572

7 Jokela R, Ahonen J, Tallgren M, Haanpää M, Korttila K. Premedication with pregabalin 75 or $150 \mathrm{mg}$ with ibuprofen to control pain after day-case gynaecological laparoscopic surgery. Br J Anaesth 2008;100(6):834-840

8 Quiney N, Cooper R, Stoneham M, Walters F. Pain after craniotomy. A time for reappraisal? Br J Neurosurg 1996;10(3):295-299

9 Kotak D, Cheserem B, Solth A. A survey of post-craniotomy analgesia in British neurosurgical centres: time for perceptions and prescribing to change? Br J Neurosurg 2009;23(5):538-542

10 Choi JB, Shim YH, Lee YW, Lee JS, Choi JR, Chang CH. Incidence and risk factors of postoperative nausea and vomiting in patients with fentanyl-based intravenous patient-controlled analgesia and single antiemetic prophylaxis. Yonsei Med J 2014;55(5):1430-1435

11 Kuo A, Wyse BD, Meutermans W, Smith MT. In vivo profiling of seven common opioids for antinociception, constipation and respiratory depression: no two opioids have the same profile. Br J Pharmacol 2015;172(2):532-548

12 White PF, Tufanogullari B, Taylor J, Klein K. The effect of pregabalin on preoperative anxiety and sedation levels: a dose-ranging study. Anesth Analg 2009;108(4):1140-1145

13 Colak S, Erdogan MO, Afacan MA, et al. Neuropsychiatric side effects due to a transdermal fentanyl patch: hallucinations. Am J Emerg Med 2015;33(3):477.e1-477.e2

14 Benyamin R, Trescot AM, Datta S, et al. Opioid complications and side effects. Pain Physician 2008;11(2, Suppl):S105-S120

15 Werner C, Kochs E, Bause H. Hoffman WE, Schulte am Esch $\mathrm{J}$. Effects of sufentanil on cerebral hemodynamics and intracranial pressure in patients with brain injury. Anesthesiology 1995;83(4):721-726

16 Pühringer F, Hörmann CH, Langmayr J, Schalow S, Benzer A. The effect of alfentanil on cerebrospinal fluid pressure in human volunteers. Eur J Anaesthesiol 1997;14(2):211-214

17 Saraswat V, Arora V. Preemptive gabapentin vs pregabalin for acute postoperative pain after surgery under spinal anaesthesia. Indian J Anaesth 2008;52:829-834

18 Lam DM, Choi SW, Wong SS, Irwin MG, Cheung CW. Efficacy of pregabalin in acute postoperative pain under different surgical categories: a meta-analysis. Medicine (Baltimore) 2015;94(46):e1944

19 Mishriky BM, Waldron NH, Habib AS. Impact of pregabalin on acute and persistent postoperative pain: a systematic review and meta-analysis. Br J Anaesth 2015;114(1):10-31

20 Shimony N, Amit U, Minz B, et al. Perioperative pregabalin for reducing pain, analgesic consumption, and anxiety and enhancing sleep quality in elective neurosurgical patients: a prospective, randomized, double-blind, and controlled clinical study. J Neurosurg 2016;125(6):1513-1522 